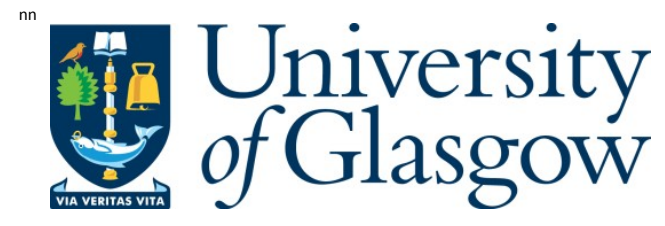

Hirose, E. et al. (2014) Mechanical loss of a multilayer tantala/silica coating on a sapphire disk at cryogenic temperatures: toward the KAGRA gravitational wave detector. Physical Review D, 90 (10). 102004 . ISSN 1550-7998

Copyright (c) 2014 A merican Physical Society

http://eprints.gla.ac.uk/100244/

Deposited on: 19 December 2014

Enlighten - Research publications by members of the University of Glasgow http://eprints.gla.ac.uk 


\title{
Mechanical loss of a multilayer tantala/silica coating on a sapphire disk at cryogenic temperatures: Toward the KAGRA gravitational wave detector
}

\author{
Eiichi Hirose $,{ }^{1}, *$ Kieran Craig, ${ }^{2}$ Hideki Ishitsuka, ${ }^{1}$ Iain W. Martin, ${ }^{2}$ Norikatsu Mio, ${ }^{3}$ Shigenori \\ Moriwaki, ${ }^{3}$ Peter G. Murray, ${ }^{2}$ Masatake Ohashi, ${ }^{1}$ Sheila Rowan, ${ }^{2}$ Yusuke Sakakibara, ${ }^{1}$ \\ Toshikazu Suzuki, ${ }^{4,1}$ Kouichi Waseda, ${ }^{5}$ Kyohei Watanabe, ${ }^{3}$ and Kazuhiro Yamamoto ${ }^{1}$ \\ ${ }^{1}$ Institute for Cosmic Ray Research, The University of Tokyo, \\ 5-1-5 Kashiwa-no-ha, Kashiwa, Chiba 277-8582, Japan \\ ${ }^{2}$ SUPA, Institute for Gravitational Research, School of Physics and Astronomy, \\ University of Glasgow, Glasgow G12 8QQ, Scotland \\ ${ }^{3}$ Photon Science Center, The University of Tokyo, \\ 2-11-16 Bunkyo-ku, Hongo, Tokyo 113-8656, Japan \\ ${ }^{4}$ High Energy Accelerator Research Organization, 1-1 Oho, Tsukuba, Ibaraki 305-0801, Japan \\ ${ }^{5}$ National Astronomical Observatory of Japan, 2-21-1 Osawa, Mitaka, Tokyo 181-8588, Japan
}

(Dated: September 20, 2014)

\begin{abstract}
We report the results of a new experimental setup to measure the mechanical loss of coating layers on a thin sapphire disk at cryogenic temperatures. Some of the authors previously reported that there was no temperature dependence of the mechanical loss from a multilayer tantala/silica coating on a sapphire disk, both before and after heat treatment, although some reports indicate that $\mathrm{Ta}_{2} \mathrm{O}_{5}$ and $\mathrm{SiO}_{2}$ layers annealed at $600^{\circ} \mathrm{C}$ have loss peaks near $20 \mathrm{~K}$. Since KAGRA, the Japanese gravitational wave detector, currently under construction, will be operated at $20 \mathrm{~K}$ and have coated sapphire mirrors, it is very important to clarify the mechanical loss behavior of tantala/silica coatings around this temperature. We carefully investigated a tantala/silica-coated sapphire disk with the new setup, annealed the disk, then investigated the annealed disk. We found that there was no distinct loss peak both before and after annealing under particular conditions. The mechanical loss for the unannealed disk at $20 \mathrm{~K}$ was about $5 \times 10^{-4}$, as previously reported, while that for the annealed disk was approximately $6.4 \times 10^{-4}$.
\end{abstract}

\section{INTRODUCTION}

As of 2014, second-generation interferometric gravitational wave detectors are being built at several locations around the world with the aim of directly detecting gravitational waves [1-3]. Their performance will be limited by fundamental noise sources such as seismic noise, quantum noise, and thermal noise. Thermal noise originates from both the suspension system and the mirror itself, and the mirror thermal noise is caused by both its substrate and coating layers. The thermal noise of mirror coatings potentially limits the sensitivity of gravitational wave detectors [4-6]. Currently, mirror coatings used in gravitational wave detectors are deposited by ion-beam sputtering and consist of alternating silica and tantala bilayers [7-9]. Investigations are underway to study the effects of doping the tantala layers with titania and optimizing the thickness of the coating layers [10-12]. Attempts have also been made to minimize socalled thermo-optic noise while cancelling the constituent thermoelastic and thermorefractive noises [13]. A direct way to reduce thermal noise is to cool the mirrors to a cryogenic temperature, and among the currently existing detectors, only KAGRA will employ cryogenic sapphire mirrors. This technology may be employed in third-generation gravitational wave detectors such as the

* hirose@icrr.u-tokyo.ac.jp
Einstein Telescope [14]. Although silicon is a candidate substrate material, sapphire has an advantage of high thermal conductivity near the operating temperature. A proof-of-concept cryogenic detector, CLIO, was built using sapphire test masses in 2005 [15-17], and it was recently shown that sapphire would be suitable for the KAGRA test mass optics [18]. The sapphire test masses are planned to be coated with a multilayer stack consisting of the dielectric materials silica and tantala. However, Martin and co-workers [19-22] and Granata et al. [23] reported that both materials have a significant mechanical loss peak near the operating temperature. Tantala single layers have a peak at $\sim 35 \mathrm{~K}$ when heat-treated at $300^{\circ} \mathrm{C}$ and $400^{\circ} \mathrm{C}[21]$. A peak develops at $20 \mathrm{~K}$ after heat treatment at $600^{\circ} \mathrm{C}$, and a peak at a similar temperature is observed in silica coatings heat-treated at $600^{\circ} \mathrm{C}$ [22]. Multilayer coatings of silica and titania-doped tantala, designed for use in advanced LIGO, exhibited a peak at $28-30 \mathrm{~K}$ after heat treatment at $400-600^{\circ} \mathrm{C}$ [23]. On the other hand, previous measurements by some of the authors did not indicate such a structure and showed that the mechanical loss of a tantala/silica multilayer coating on a sapphire disk was temperature-independent [24]. One difference between the two studies is the specific detail of the heat treatments applied to the coatings studied. Therefore, using an identical disk, we decided to investigate the behavior of the mechanical loss of the coated disk carefully as a function of temperature, then anneal the disk and remeasure it. 
TABLE I. Annealing conditions.

\begin{tabular}{cccc}
\hline \hline Ramp rate & Baking time & Temperature & Cooling rate \\
$2^{\circ} \mathrm{C} / \mathrm{min}$ & $10 \mathrm{hrs}$ & $500^{\circ} \mathrm{C}$ & Naturally \\
\hline \hline
\end{tabular}

\section{EXPERIMENT}

We performed ring-down measurements in a newly developed setup at ICRR on coated and uncoated sapphire disks of $100 \mathrm{~mm}$ diameter and $0.5 \mathrm{~mm}$ thickness. The disks were manufactured by Shinkosha [25], and coated and annealed by one of the authors at NAOJ. The coating consists of 30 layers of the dielectric materials $\mathrm{Ta}_{2} \mathrm{O}_{5}$ and $\mathrm{SiO}_{2}$ as expressed by sapphire $\left|(\mathrm{HL})^{15} \mathrm{~L}\right|$ air, where $\mathrm{H}$ and L denote each layer of the high-index material (tantala) and low-index material (silica) of a quarter wavelength for a $1064 \mathrm{~nm}$ laser. Taking into account each refractive index, the total physical thickness of the coating layers is approximately $4.5 \mu \mathrm{m}$.

When an oscillating system follows a exponential decay $\sim e^{-\lambda t}$ in its amplitude over a period of time $t$, where $\lambda$ is the decay constant or inverse of the time constant, the quality factor $Q$ is defined as

$$
Q=\frac{\pi f}{\lambda},
$$

where $f$ is the frequency of the system's resonant mode in which we are interested. Mechanical loss is defined by $\phi=1 / Q$. The mechanical loss of the coating is extracted by taking the difference in the loss between coated disk and uncoated disk, correcting for the ratio $C$ of energy stored in the coating to that in the substrate [26],

$$
\phi_{\text {coating }}=C\left(\frac{1}{Q_{\text {coated }}}-\frac{1}{Q_{\text {disk }}}\right),
$$

where $C$ is approximately $(1 / 3)\left(d_{s} / d_{c}\right)\left(Y_{s} / Y_{c}\right)$ and $d_{s}$, $d_{c}, Y_{s}$, and $Y_{c}$ are the thickness and Young's modulus of the substrate and the coating layers, respectively. Their values can be found in [24]. $Q_{\text {coated }}$ and $Q_{\text {disk }}$ are the quality factors of the coated and uncoated disk, respectively. Strictly speaking, EQ.(2) does not give the pure mechanical loss of the coating since there should be other losses such as thermoelastic loss [27]. As discussed later, however, the thermoelastic loss in our system will be negligible at the temperatures of interest. We first determined the quality factor of the as-deposited coating, then did the same after the coated disk was annealed. The annealing was performed in air under the conditions shown in TABLE I.

The clamp mechanism made of copper used to fix the sapphire disk to the central vibrational node is the same as that used in [24]. The support system shows a loss, but the loss is negligible when the quality factor $Q$ is smaller than $10^{6}[24]$. A driver, used to excite the resonant modes of the disk, is also attached to the clamp (FIG. 1). The disk-clamp system was placed in a cryostat equipped with

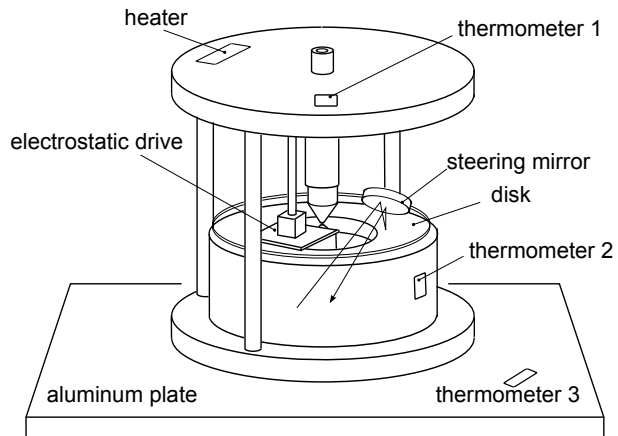

FIG. 1. The sapphire disk is clamped at the center and an electrode is bolted onto the top plate so that the clearance between the disk and the electrode is adjustable. The body, made of copper, has a heater on the top plate to accurately control the disk temperature, and three thermometers are attached to monitor the disk temperature. The body is placed inside a cryostat, and the motion of the disk is monitored using an optical lever.

a dry pump, a turbo pump, and a pulse tube cryocooler whose refrigerant is helium. The cryostat can cool the system to about $15 \mathrm{~K}$. A sinusoidal wave with a peak voltage of $2-3 \mathrm{~V}$, generated using an Agilent 33220A signal generator with $200-300 \mathrm{~V}$ DC offset produced by a Matsusada HPMQ-1P high voltage supply, was injected into the electrostatic drive plate to excite the disk. The clearance between the disk and the electrode was set to about $0.5-1 \mathrm{~mm}$.

To extract the motion of the disk, we constructed an optical lever system comprising a $2 \mathrm{~mW}$ He-Ne laser (Neoark HN-510P) and a Hamamatsu S4349 quadrant Si PIN photodiode (QPD, hereafter). The system comprising the laser and QPD were placed outside the cryostat, and the laser beam was passed through an optical window on the cryostat to monitor the motion of the disk. A steering mirror was mounted inside the cryostat as part of the optical lever system.

A heater on top of the clamp was used to control the disk temperature. For measurements below about $50 \mathrm{~K}$, we controlled the temperature of the disk by changing the heater power, while above $50 \mathrm{~K}$ we switched off the cryocooler unit to allow the disk temperature to increase naturally. Since the clamp system has a temperature gradient from the low-temperature baseplate to the hightemperature heater on the top plate, we calibrated the disk temperature by attaching a thermometer to a blank sapphire test disk and recording the disk temperature as a function of both the heater power and the other measured temperatures. This disk was not used for mechanical loss measurements since the attachment would have caused high loss. We used DT-670-SD Lake Shore silicon diode temperature sensors and a Yokogawa DX230-2-1 recorder for data storage. It was established that the disk temperature was the same as that of the top plate in the entire temperature range, but there was some devi- 


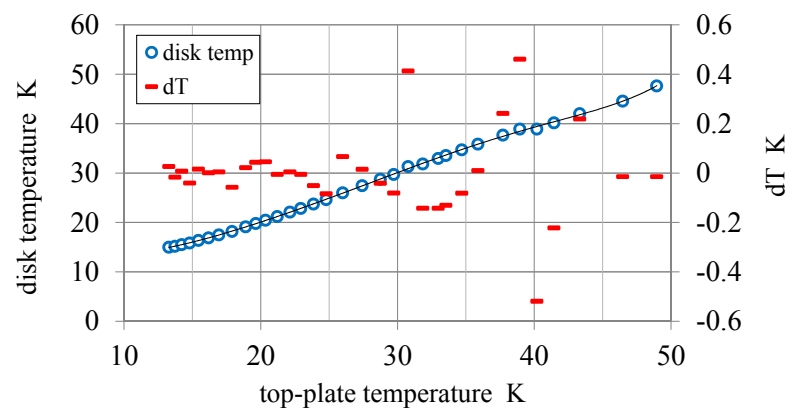

FIG. 2. The disk temperature was calibrated against the topplate temperature when the heater was switched on. We fitted the result with a polynomial function, and $d T$ is the deviation between the measured points and the polynomial function. Below $30 \mathrm{~K}, d T$ is less than $0.1 \mathrm{~K}$.

ation when the heater was switched on (i.e., below $50 \mathrm{~K}$ ). FIG. 2 shows the disk temperature as a function of the top-plate temperature. The measured points were fitted and the obtained polynomial function was used to deduce the disk temperature during the ring-down measurement. The deviation between the measured points and the polynomial function is also shown as $d T$ in FIG. 2, and it is less than $0.1 \mathrm{~K}$ below $30 \mathrm{~K}$, in the temperature range of interest.

FIG. 3 shows the output of the QPD obtained using an Advantest R9211C spectrum analyzer with a disk temperature of $28 \mathrm{~K}$ when we injected a $527 \mathrm{~Hz}$ sinusoidal signal into the electrode to excite a quadrupole mode. The frequency decreases as the disk temperature increases owing to the change in the Young's modulus of the substrate. To ensure that we were actually looking at the targeted mode, the signal from the QPD was investigated. 'Dark' stands for the case when there is no laser light entering the QPD. The blue line in the figure shows the frequency spectrum of a typical excited resonant mode of the disk. The fact that it is considerably above the dark noise in these photodiodes demonstrates that the observations were indeed the oscillation of the disk. By injecting an off-resonant frequency signal, the peak of the quadrupole mode disappears from the blue curve.

The ring-downs were measured by passing the QPD signal through a Stanford Research Systems SR530 lockin amplifier and recording the lock-in signal using an EasySYNC DSIM12 data logger. At each temperature, the ring-down measurement was repeated several times. The quality factor was extracted by fitting exponential decay curves to the measured ring-downs. Pressure inside the cryostat remained between $10^{-7} \mathrm{mbar}$ and $10^{-5} \mathrm{mbar}$ up to $150 \mathrm{~K}$, and increased to $10^{-4}$ mbar order above this temperature, but it is still sufficiently low to ignore the effects of gas damping [28].

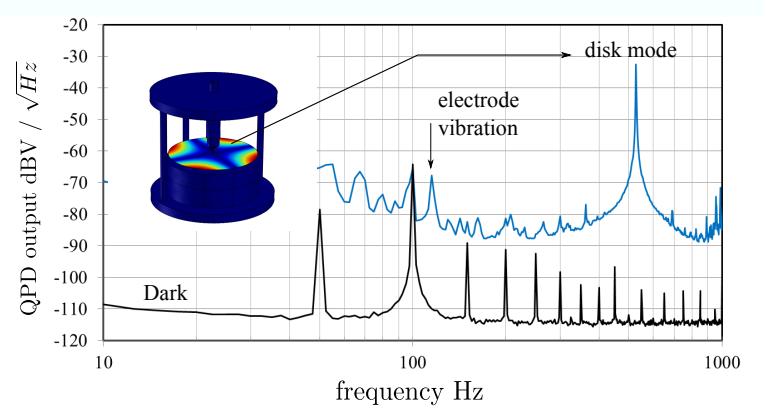

FIG. 3. Spectrum analyzer output of QPD signal at $28 \mathrm{~K}$. 'Dark' represents the case when no laser light hits the QPD, where the harmonics from the power line are apparent. The blue line shows the frequency spectrum of a typical excited resonant mode of the disk. The excited quadrupole mode is the only strong signal in the frequency range. The electrode bolted onto the top plate vibrates and the motion appears in the signal.

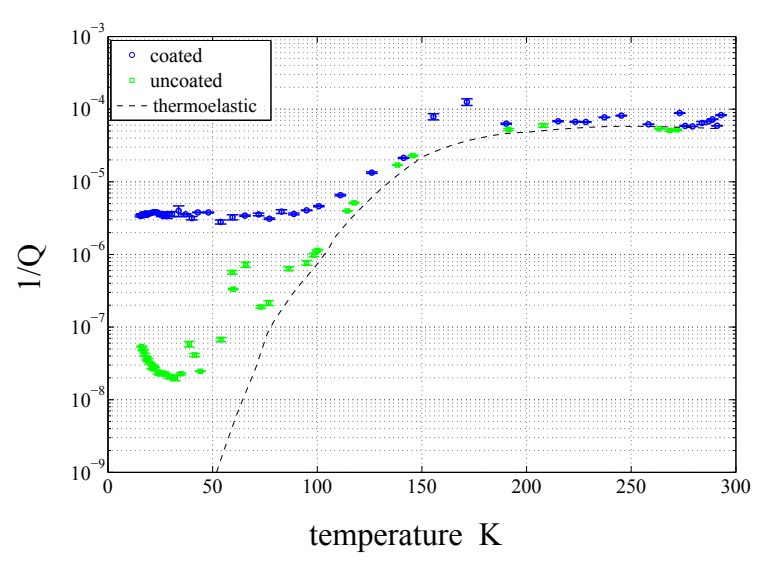

FIG. 4. Blue circles and green squares indicate the unannealed coated and uncoated disks, respectively. The black dashed line shows the thermoelastic loss. The quality factor was measured three times at each temperature, and the points and error bars in the plot represent the average and standard deviation, respectively.

\section{RESULTS}

FIG. 4 shows $1 / Q_{\text {coated }}$ and $1 / Q_{\text {uncoated }}$ in terms of the disk temperature, which correspond to the results for an as-deposited unannealed disk (blue circles) and an uncoated disk (green squares), respectively. Each point is the mean of three measurements and the error bar is twice the standard deviation. Also, the thermoelastic loss in the sapphire substrate is shown as a dashed line [29,30] on the basis of the properties in the literature [31]. The thermoelastic loss is dominant above about $100 \mathrm{~K}$ and the thermoelastic effect is negligible below $50 \mathrm{~K}$. There are two distinct loss peaks for the uncoated sapphire disk at approximately $40 \mathrm{~K}$ and $65 \mathrm{~K}$. These temperatures giving 
attenuation peaks are consistent with the measurements reported in [32], in which a peak around $\sim 30-40 \mathrm{~K}$ is associated with a phonon-phonon coupling process while a peak around $\sim 50-80 \mathrm{~K}$ is associated with a relaxation process. We observed the start of another attenuation increase toward $10 \mathrm{~K}$, but we could not reach the required temperature in our setup. For the unannealed coated sapphire disk, there was no apparent structure below $100 \mathrm{~K}$. Since below $50 \mathrm{~K}, 1 / Q_{\text {uncoated }}$ is generally about two orders of magnitude smaller than both $1 / Q_{\text {coated }}$ and the standard deviation of the results for the coated disk, one can approximate the mechanical loss of the coating as $\phi_{\text {coated }}=C / Q_{\text {coated }}$, and this definition is used in the following discussion.

FIG.5 shows the mechanical loss of the annealed coated stack below $50 \mathrm{~K}$. The ring-down measurement was performed five times for each point and each mark represents the mean of five measurements. The error bar is twice the standard deviation as before. The plot shows the loss of the annealed disk at three different frequencies, $521 \mathrm{~Hz}$ (mode 1), $1204 \mathrm{~Hz}$ (mode 2), and $4594 \mathrm{~Hz}$ (mode 4), and the loss of the unannealed disk is also shown (blue circles). The black squares are the results of Yamamoto et al.(mode 1) [24]. The mode shapes corresponding to these frequencies, calculated by COMSOL [33], are also shown in the figure. When the coating mechanical loss for these modes was calculated, correction factors were used owing to the fact that the coating aperture is $90 \%$ of the disk's diameter. The strain energy ratio $r_{E}=E_{1} / E_{\text {total }}$ of the thin disk was calculated, where $E_{1}$ and $E_{\text {total }}$ are the strain energy inside a diameter of $90 \mathrm{~mm}$ and that of the entire disk, respectively. $r_{E}$ for modes 1,2 , and 3 are $0.8661,0.7708$, and 0.5842 , respectively, and $C$ in EQ.(2) was replaced with $C / r_{E}$ so as not to underestimate the coating mechanical losses. The loss at an additional frequency of $2115 \mathrm{~Hz}$ (mode 3) was also measured, for which a loss peak centered around $33 \mathrm{~K}$ was observed. However, when measurements of the loss of this mode were repeated with the compressor turned off, the loss at this temperature decreased to $\sim 6 \times 10^{-4}$, suggesting that the apparent loss peak was related to the effects of the vibration of the compressor. It was, however, not possible to remeasure the loss of this frequency over the entire temperature range with the compressor turned off because of the rate at which the temperature increased. Measurements of the unannealed disk gave a temperature independent mechanical loss of $5 \times 10^{-4}$ while a temperatureindependent loss of $\sim 4 \times 10^{-4}$ was reported in [24]. The results for the annealed disk also did not show any apparent peaks. The mechanical loss for mode 1 is greater than that for the unannealed disk as reported in [21] and varies between $6 \times 10^{-4}$ and $7 \times 10^{-4}$ in the temperature range. The measurement by Yamamoto et al. [24] did not have as fine temperature steps as those in our measurements, thus it was not clear whether any structure existed in their study. It was confirmed that the multilayer tantala/silica coating had no dissipation peak near the KAGRA's operating temperature of 20 Kelvin.

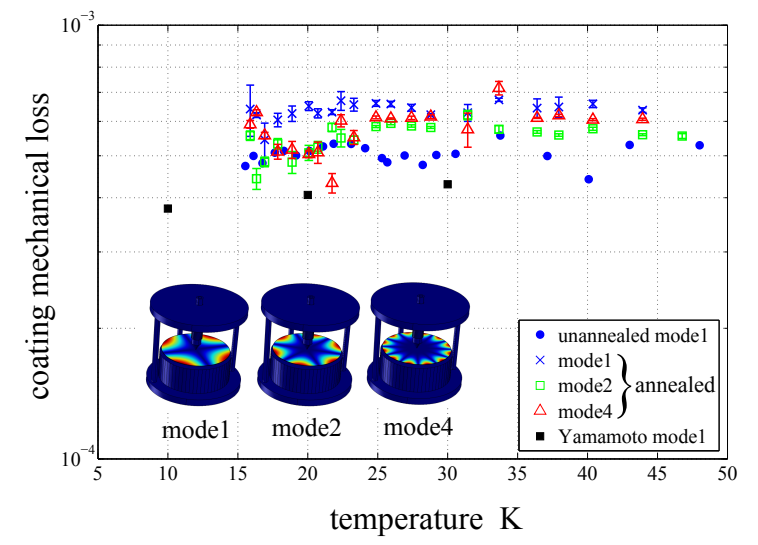

FIG. 5. Blue circles show the mechanical losses of the unannealed coated disk (mode 1), and black squares show those in [24]. The other results are for the annealed coated disk at three different frequencies: $521 \mathrm{~Hz}$ (mode 1, red crosses), $1204 \mathrm{~Hz}$ (mode 2, green squares), and $4594 \mathrm{~Hz}$ (mode 4, cyan triangles). The unannealed disk reproduced the same temperature-independent behavior as previously observed, and the annealed disk also did not have any apparent peaks below $50 \mathrm{~K}$.

TABLE II. Results from Martin et al. [21, 22]. T' shows the temperature giving the Debye peak at $520 \mathrm{~Hz}$ if the process followed the Arrhenius formula.

\begin{tabular}{ccccc}
\hline \hline material & $\mathrm{T}_{\text {peak }}[\mathrm{K}]$ & $\mathrm{E}_{a}[\mathrm{meV}]$ & $\tau_{0}\left[10^{-14} \mathrm{~s}\right]$ & $\mathrm{T}^{\prime}[\mathrm{K}]$ at $520 \mathrm{~Hz}$ \\
$\mathrm{Ta}_{2} \mathrm{O}_{5}$ & 20 & $35.6 \pm 2.5$ & $(99 \pm 5)$ & $21.1 \pm 1.6$ \\
$\mathrm{Ta}_{2} \mathrm{O}_{5}$ & 35 & $66 \pm 10$ & $(9.4 \pm 0.9)$ & $35.0 \pm 5.5$ \\
$\mathrm{SiO}_{2}$ & 20 & $32.1 \pm 2.9$ & $(18 \pm 1)$ & $17.5 \pm 1.7$ \\
\hline \hline
\end{tabular}

\section{DISCUSSION}

Internal friction mechanisms may be thought of as relaxation processes [32] and can be successfully modeled using

$$
\frac{1}{Q}=\Delta \frac{\omega \tau}{1+(\omega \tau)^{2}},
$$

where $\omega=2 \pi f, \Delta$ is the intensity of the relaxation process, and $\tau$ is the relaxation time. The loss has a peak when $\omega \tau=1$, called the Debye peak. For relaxation processes associated with the movement of atoms from one location to another, $\tau$ is given by the Arrhenius formula $\tau=\tau_{0} \exp \left[E_{a} /\left(k_{B} T\right)\right]$, where $E_{a}$ is the activation energy, $\tau_{0}$ is the rate constant, $k_{B}$ is the Boltzmann constant, and $T$ is the absolute temperature. The activation energy can be found by measuring the temperature that gives the Debye peak at different frequencies. Martin and co-workers found the Debye peak at $20 \mathrm{~K}$ for both tantala and silica, and they measured the activation energies and rate constants $[21,22]$ to be those listed in TABLE II. The final column in the table, T', shows 

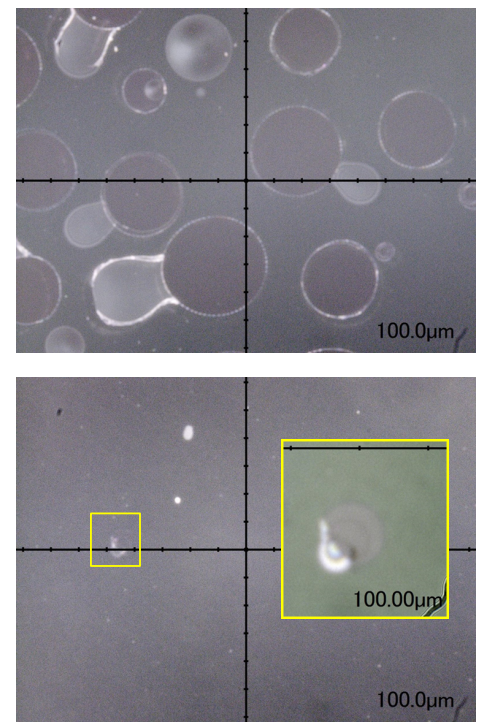

FIG. 6. Dark field images of disks annealed at $600^{\circ} \mathrm{C}$ (upper) and $500^{\circ} \mathrm{C}$ (lower). The latter disk was investigated in this study, and a zoomed bright-field image is also shown. Although the defect density is very small, in the disk annealed at $500^{\circ} \mathrm{C}$, similar defects to those appearing in the upper disk were observed.

the temperature giving the Debye peak at $520 \mathrm{~Hz}$ if the process followed the Arrhenius formula. The fact that no peaks were observed at these temperatures indicates that the internal friction mechanism in the multilayer coating will be more complicated than the above model. It is interesting to note, however, that Granata et al. [23] reported that the loss of a coating deposited in the same run as one of the advanced LIGO mirrors exhibited a peak at $\sim 28-30 \mathrm{~K}$. They use a titania-doped tantala/silica coating heat-treated at $\sim 500^{\circ} \mathrm{C}$. The final coating of the KAGRA detector has not yet been determined and the coating on the sapphire disk in this report is by no means the final one. A high reflectivity coating will require thicker coating layers, and the number of layers is likely to be more than 40 . Regarding the annealing regime used for KAGRA, a lower temperature and a shorter annealing time would be preferable unless strictly necessary to meet the required optical absorption and reflectance. With this in mind, it is probably reasonable to conclude that a somewhat greater coating mechanical loss was observed than would have occurred for the test masses. Prior to the annealing under the conditions in TABLE I, an identical disk from the same batch was first annealed at $600^{\circ} \mathrm{C}$ for $24 \mathrm{hrs}$. However, in this case, the coating layers were completely destroyed. FIG.6 shows dark-field images of the disk obtained by a Keyence VHX-1000 microscope after annealing at $600^{\circ} \mathrm{C}$ (upper) and $500^{\circ} \mathrm{C}$ (lower). The upper image shows a large number of circular defects at which the coating layers have peeled off. Although such a distinct feature could not be seen in the disk annealed at $500^{\circ} \mathrm{C}$, several smaller defects were found. Considering the importance of coatings to the sensitivity of gravitational wave detectors, this research must be continued using coatings with more similar specifications to those of the final test mass mirrors.

\section{CONCLUSION}

A setup was developed to measure the mechanical loss of coatings. The mechanical loss of a multilayer coating of $\mathrm{Ta}_{2} \mathrm{O}_{5} / \mathrm{SiO}_{2}$ on a previously measured sapphire disk was quantified with a finer temperature resolution. Then, the disk was annealed under various conditions and remeasured to observe any effect on the mechanical loss of the coating layers. The same result was reproduced by Yamamoto et al. [24] for an unannealed disk, which indicates that the mechanical loss of the coating was temperature-independent between $15 \mathrm{~K}$ and $50 \mathrm{~K}$. For the annealed disk, there was no clear peak in the measured temperature range.

Although the previous results contained the results for heat-treated disks, details of the heating regime were not reported. The measured mechanical loss at $20 \mathrm{~K}$ for the unannealed disk $\left(\sim 5 \times 10^{-4}\right)$ was slightly larger than that measured in $[24]\left(\sim 4 \times 10^{-4}\right)$, while that for the annealed disk was $\sim 6.4 \times 10^{-4}$. This is significantly lower than the loss of the advanced LIGO coating at $20 \mathrm{~K}$ recently reported by Granata et al. [23]. However, their coating was titania-doped and the heat treatment was slightly different. Understanding the reasons for these differences in coating loss is an important area of future work. Also, since it is likely that the final coating for the KAGRA detector will differ in several aspects from the one measured in this experiment, it will be essential to verify the mechanical loss of the coating layers of the test mass mirrors in the near future.

\section{ACKNOWLEDGMENTS}

The authors would like to thank colleagues in the KAGRA collaboration, especially staff members of the Gravitational Wave Office at the Institute for Cosmic Ray Research, the University of Tokyo, for their support. We also thank Matt Abernathy for his helpful comments. This work was supported by the Joint Research Program at ICRR and the ELiTES program. EH thanks Takanori Sekiguchi and Chen Dan for their help. We are grateful for the financial support provided by SUPA, STFC (grant number ST/L000946/1), and the University of Glasgow. We would like to thank our colleagues in the LSC and Virgo collaborations and within SUPA for their interest in this work. This paper has the document numbers JGW-P1402393 and LIGO-P1400106. 
[1] LIGO Laboratory, http://www.ligo.caltech.edu/.

[2] Virgo, https://wwwcascina.virgo.infn.it/.

[3] KAGRA, http://gwcenter.icrr.u-tokyo.ac.jp/.

[4] G. Harry et al., Class. Quantum Grav. 19, 897 (2002).

[5] G. Harry et al., Appl. Opt. 45, 1569 (2006).

[6] G. Harry et al., Class. Quantum Grav. 24, 405 (2007).

[7] S. Penn et al., Class. Quantum Grav. 20, 2917 (2003).

[8] D. Crooks et al., Class. Quantum Grav. 21, 1059 (2004).

[9] D. Crooks et al., Class. Quantum Grav. 23, 4953 (2006).

[10] R. Flaminio et al., Class. Quantum Grav. 27, 084030 (2010).

[11] A. Villar et al., Phys. Rev. D 81, 122001 (2010).

[12] N. Kondratiev et al., Phys. Rev. D 84, 022001 (2011).

[13] M. Evans et al., Phys. Rev. D 78, 102003 (2008).

[14] Einstein Telescope, http://www.et-gw.eu/.

[15] M. Ohashi et al., Class. Quantum Grav. 20, 599 (2003).

[16] S. Miyoki et al., Class. Quantum Grav. 21, 1173 (2004).

[17] T. Uchiyama et al., Phys. Rev. Lett. 108, 141101 (2012).

[18] E. Hirose et al., Phys. Rev. D 89, 062003 (2014).

[19] I. Martin et al., Class. Quantum Grav. 25, 055005 (2008).
[20] I. Martin et al., Class. Quantum Grav. 26, 155012 (2009).

[21] I. Martin et al., Class. Quantum Grav. 27, 225020 (2010).

[22] I. Martin et al., Class. Quantum Grav. 31, 035019 (2014).

[23] M. Granata et al., Opt. Lett. 38, 5268 (2013).

[24] K. Yamamoto et al., Phys. Rev. D 74, 022002 (2006).

[25] Shinkosha Co., Ltd, http://www. shinkosha.com/.

[26] L. Landau and E. Lifshitz, Theory of Elasticity (Pergamon, 1986).

[27] M. Fejer et al., Phys. Rev. D 70, 082003 (2004).

[28] F. Blom et al., J. Vac. Sci. Technol.. 10, 19 (1992).

[29] D. Blair and J. Ferreirinho, Phys. Rev. Lett. 49, 375 (1982).

[30] C. Zener, Phys. Rev. 53, 230 (1938).

[31] Y. Touloukian et al., Thermophysical Properties of Matter (New York IFI/Plenum, 1970).

[32] V. Braginsky, V. Mitrofanov, and V. Panov, Systems with Small Dissipation (The University of Chicago Press, 1985).

[33] COMSOL, http://www.comsol.com/. 